\title{
An anomalous left coronary artery with a malignant course: coronary angiography and myocardial perfusion imaging with computed tomography
}

\author{
M.A. de Graaf • A.R. van Rosendael $\cdot$ L.J. Kroft $\cdot$ H.W. Vliegen $\cdot$ M.G. Hazekamp $\cdot$ J.J. Bax $\cdot$ A.J. Scholte
}

Published online: 7 January 2016

(C) The Author(s) 2016. This article is published with open access at Springerlink.com

A 41-year old woman presented at the emergency department because of collapse during running. Coronary computed tomography angiography (CTA) showed no atherosclerosis (Fig. 1). However, as demonstrated by the double oblique view of the sinus of Valsalva in Panel A and 3D-rendering of Panel B, the left coronary artery (LCA) originated from the right coronary sinus. The LCA has an acute angle and a 'slitlike' ostium which can collapse in a valve-like manner during exercise. The incidence of this finding on CTA ranges from $0.7-6.6 \%[1,2]$. Adenosine stress CT myocardial perfusion imaging (CTP) was performed. Panel C demonstrates a sub-endocardial perfusion defect in the LCA region (white arrows). Panel D represents the polar map of the transmural perfusion ratio. Surgical unroofing was performed successfully and no further events have occurred [3]. In patients

Electronic supplementary material The online version of this article (doi:10.1007/s12471-015-0788-0) contains supplementary material, which is available to authorized users.

A.J. Scholte $(\bowtie) \cdot$ M.A. de Graaf · A.R. van Rosendael · H.W. Vliegen · J.J. Bax

Department of Cardiology, Leiden University Medical Center, Albinusdreef 2,

2333 ZA Leiden, The Netherlands

e-mail: m.a.de graaf@lumc.nl

M.A. de Graaf · A.R. van Rosendael

The Interuniversity Cardiology Institute of the Netherlands,

Utrecht, The Netherlands

L.J. Kroft

Department of Radiology, Leiden University Medical Center, Leiden, The Netherlands

M.G. Hazekamp

Department of Cardiothoracic Surgery, Leiden University

Medical Center,

Leiden, The Netherlands presenting with collapse a malignant coronary anomaly can be observed. CTP can subsequently be performed to detect myocardial ischaemia.

\section{Disclosures}

Michiel A. de Graaf is supported by a research grant from the Interuniversity Cardiology Institute of the Netherlands
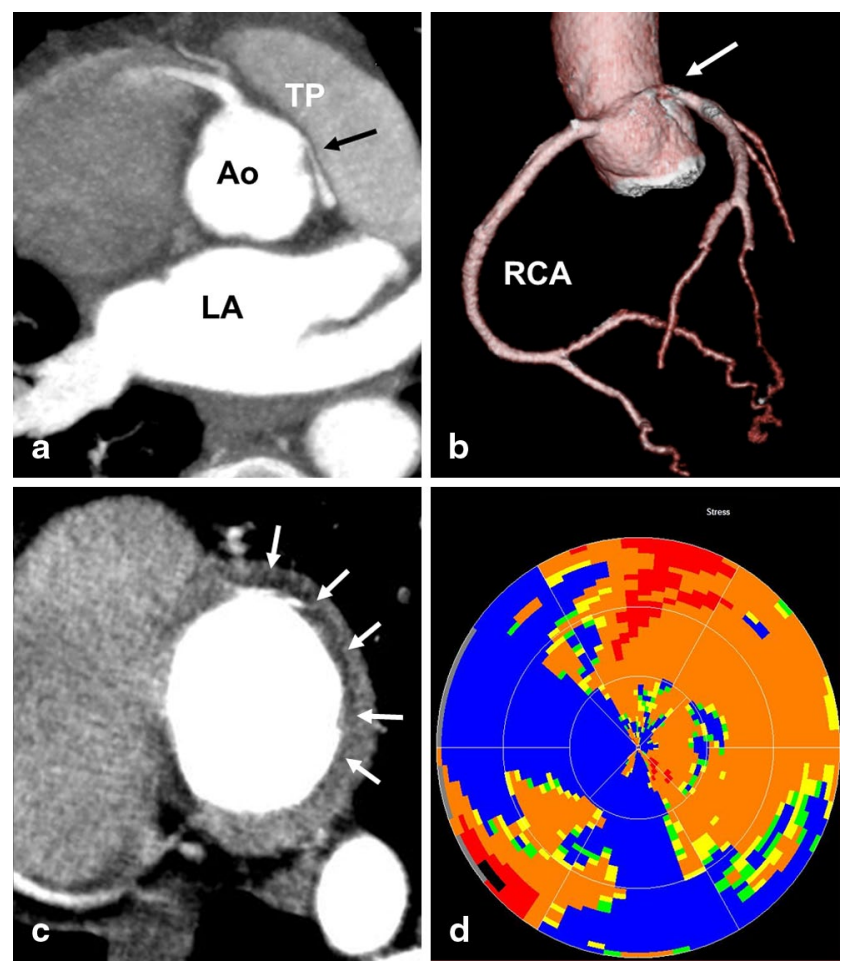

Fig. 1 Computed tomography coronary angiography and myocardial perfusion imaging 
(ICIN, Utrecht, the Netherlands). The Department of Cardiology received research grants from Biotronik, Medtronic, Boston Scientific Corporation, St Jude Medical, Lantheus Medical Imaging and GE Healthcare.

Open Access This article is distributed under the terms of the Creative Commons Attribution License which permits any use, distribution, and reproduction in any medium, provided the original author(s) and the source are credited.

\section{References}

1. Taylor AJ, Rogan KM, Virmani R. Sudden cardiac death associated with isolated congenital coronary artery anomalies. J Am Coll Cardiol. 1992;20:640-7.
2. Zhang LJ, Yang GF, Huang W, Zhou CS, Chen P, Lu GM. Incidence of anomalous origin of coronary artery in 1879 Chinese adults on dual-source CT angiography. Neth Heart J. 2010;18:466-70.

3. Nguyen AL, Haas F, Evens J, Breur JM. Sudden cardiac death after repair of anomalous origin of left coronary artery from right sinus of Valsalva with an interarterial course: case report and review of the literature. Neth Heart J. 2012;20:463-71. 